\title{
Neutrophil enhancement of Pseudomonas aeruginosa biofilm development: human F-actin and DNA as targets for therapy
}

\begin{abstract}
Correspondence
Quinn M. Parks

parksq@njc.org
\end{abstract}

Received 9 August 2008

Accepted 10 November 2008

\author{
Quinn M. Parks, ${ }^{1}$ Robert L. Young, ${ }^{2}$ Katie R. Poch, ${ }^{1}$ Kenneth C. Malcolm, ${ }^{1}$ \\ Michael L. Vasil ${ }^{3}$ and Jerry A. Nick ${ }^{1,2}$
${ }^{1}$ Department of Medicine, National Jewish Health, 1400 Jackson, Denver, CO, USA
${ }^{2}$ Division of Pulmonary Science and Critical Care Medicine, University of Colorado, 4200 E. 9th Ave, Denver, CO, USA
${ }^{3}$ Department of Microbiology, University of Colorado Denver Anschutz Medical Campus, 12800 E. 19th Ave, Aurora, CO, USA

\begin{abstract}
In the cystic fibrosis (CF) airway, chronic infection by Pseudomonas aeruginosa results from biofilm formation in a neutrophil-rich environment. We tested the capacity of human neutrophils to modify early biofilm formation of $P$. aeruginosa strain PAO1, and an isogenic CF strain isolated early and years later in infection. In a static reactor, $P$. aeruginosa biofilm density of all strains was enhanced at $24 \mathrm{~h}$ in the presence of neutrophils, with the greatest relative increase associated with the lowest inoculum of $P$. aeruginosa tested. Previously, neutrophil-induced biofilm enhancement was shown to largely result from the incorporation of F-actin and DNA polymers into the bacterial biofilm. This finding was advanced by the comparison of biofilm enhancement from intact unstimulated neutrophils and from lysed or apoptotic neutrophils. Apoptotic neutrophils, with an intact cell membrane, were unable to contribute to biofilm enhancement, while lysed neutrophils evoked a similar response to that of intact cells. Using F-actin and DNA as targets, the capacity of negatively charged poly(amino acids) to disrupt, or prevent, early biofilm formation was tested. Anionic poly(aspartic acid) effectively prevented or disrupted biofilm formation.

Combination of poly(aspartic acid) with DNase resulted in a synergistic increase in biofilm disruption. These results demonstrate that the presence of dying neutrophils can facilitate the initial stages of biofilm development by low inocula of $P$. aeruginosa. Neutrophil F-actin represents a potential new therapeutic target for disruption of pathogenic biofilms.
\end{abstract}

\section{INTRODUCTION}

Cystic fibrosis (CF) lung disease features persistent neutrophil accumulation within the airways from the time of infancy (Khan et al., 1995). Inhalation of aerosolized Pseudomonas aeruginosa occurs frequently from environmental exposures, including dirt and contaminated water (Baumgartner \& Grand, 2006; Burns et al., 2001; Khan et al., 2007). While eradication of $P$. aeruginosa is possible in childhood, persistent infection eventually occurs in over $80 \%$ of adult CF patients. Chronic $P$. aeruginosa infection is clearly linked with increased morbidity and mortality in CF (Emerson et al., 2002; Parad et al., 1999; Schaedel et al., 2002), and is associated with phenotypic and genetic changes of the bacteria within the airway, including the formation of biofilms (Drenkard \& Ausubel, 2002; Ernst et al., 1999;

Abbreviations: CF, cystic fibrosis; CV, crystal violet; SEM, scanning electron microscopy.

Supplementary figures are available with the online version of this paper.
Spencer et al., 2003). In classical models, bacterial biofilms are surface-attached communities of cells encased within a self-produced extracellular polysaccharide matrix, often associated with mucoidy (Costerton et al., 1999; Klausen et al., 2003). However, biofilms in the CF airway are not typically surface-associated, but rather are small aggregates of bacteria suspended in the highly tenacious CF sputum (Singh et al., 2000; Sriramulu et al., 2005).

Persistent accumulation of neutrophils in the CF airways, combined with ineffective clearance, leads to the uncoordinated release of DNA, actin and granule proteins from necrotic cells. This process results in mucus highly enriched with these products, all of which are clearly implicated in the pathogenesis of CF lung disease (Balfour-Lynn, 1999; Khan et al., 1995; Lethem et al., 1990; Perks \& Shute, 2000; Roum et al., 1993; Sheils et al., 1996). Previously, we have reported that the level of early biofilm formation by $P$. aeruginosa strain PAO1 is increased in the presence of neutrophils (Walker et al., 2005). A principal mechanism 
of the enhancement is filaments of neutrophil-derived DNA and F-actin, which provide a ready framework for $P$. aeruginosa biofilm development (Walker et al., 2005).

The F-actin and DNA framework formed following neutrophil necrosis is an attractive potential target for therapeutic intervention, as disrupting this scaffold could limit or decrease the integrity of an early $P$. aeruginosa biofilm. Negatively charged strands of DNA when present with F-actin are linked via positively charged histones and cations (Tang et al., 2005). Recently it has been shown that polyanionic peptides have the capacity to disassociate DNA-histone, as well as F-actin-histone complexes, and disaggregate F-actin bundles (Tang \& Janmey, 1996; Tang et al., 2005). These findings suggest that a DNA-based biofilm that has incorporated F-actin could be disrupted by anionic polymers via electrostatic competition. Further, the addition of these anionic polymers could cooperatively enhance the activity of current therapies such as DNase.

\section{METHODS}

Bacterial strains, media and culture conditions. All $P$. aeruginos strains were originated from stock plates of Luria broth (LB) agar, grown overnight in RPMI 1640 supplemented with L-glutamine and $2 \%$ heat-inactivated (human) platelet-poor plasma (HIPPP) at $37{ }^{\circ} \mathrm{C}$ with moderate shaking (225 r.p.m.) overnight, and frozen down to achieve consistent growth phase. All assays utilized bacteria grown overnight from the frozen stock in RPMI $+2 \%$ HIPPP at $37{ }^{\circ} \mathrm{C}$. Cultures were then equilibrated in a Beckman DU 640 spectrophotometer to an $\mathrm{OD}_{650}$ of $0.30\left(5 \times 10^{8}\right.$ c.f.u. $\left.\mathrm{ml}^{-1}\right)$. Serial 10 -fold dilutions of the cultures were performed in RPMI. Strains utilized were PAO1 (from B. H. Iglewski, University of Rochester, New York, USA) and an early and late strain isolated from a single patient days after initial infection, and following years of infection (Ogle et al., 1987).

Neutrophil isolation. Neutrophils for all assays were isolated from healthy volunteers by the plasma Percoll method as previously described (Haslett et al., 1985).

Crystal violet (CV) biofilm assay. Donor neutrophils were

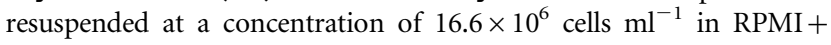
$2 \%$ HIPPP in a Nunc round-bottom 96-well plate. The cellular control was a plate identical to the test plate, save for the absence of neutrophils. Dilutions of bacteria were tested at a $1: 1$ ratio to a $1: 0.001$ ratio, thus final concentrations of bacteria ranged from $16.6 \times 10^{6}$ to $16.6 \times 10^{3}$ c.f.u. $\mathrm{ml}^{-1}$. A Nunc TSP lid system, which comprises a 96-well plate lid with pegs that extend into each well, was then placed on a 96-well plate. This assembly was then incubated for $24 \mathrm{~h}$ with rocking (3-4 oscillations $\min ^{-1}$ ) at $37^{\circ} \mathrm{C}$. At $24 \mathrm{~h}$, the peg lids with bacterial biofilms were removed and rinsed for $2 \mathrm{~min}$ in sterile normal saline to remove non-adherent bacteria and cellular debris. The peg lid was airdried for $5 \mathrm{~min}$, then fixed for $10 \mathrm{~min}$ in $100 \%$ ethanol. The ethanol was evaporated for $5 \mathrm{~min}$ on the bench top, then biofilms were stained in $1 \% \mathrm{CV}$ for $15 \mathrm{~min}$. Excess CV was removed by two rinses in normal saline. Decolorization took place in a Nunc 96-well flat-bottom plate with $200 \mu 100 \%$ methanol per well for $45 \mathrm{~min}$. Readings were taken in a $\mu$ Quant plate reader using the KCjunior v1.403 software at $550 \mathrm{nM}$ (BioTek Instruments). Values within control samples were averaged prior to comparison of each test well.

Amino acid preparation. Pure amino acid chains of multiple lengths were initially diluted in PCR grade $\mathrm{dH}_{2} \mathrm{O}$ to $1 \mathrm{M}$, then serially diluted in normal saline. Peptides used were poly(L-aspartic acid) (Sigma) and poly(serine) (MP Biomedicals). No further purification was performed. Pulmozyme (DNase) (Genetech; NDC 50242-010040) was added at final concentrations of $33 \mu \mathrm{g}$ DNase $\mathrm{ml}^{-1}$ or $8.25 \mu \mathrm{g}$ DNase $\mathrm{ml}^{-1}$. Protease inhibitors were used at final concentrations of $2.56 \mu \mathrm{g}$ leupeptin $\mathrm{ml}^{-1}, 1.03 \mu \mathrm{g}$ pepstatin $\mathrm{A} \mathrm{ml}^{-1}$ and $2.56 \mu \mathrm{g}$ aprotinin $\mathrm{ml}^{-1}$.

Quantification of viable bacteria within biofilm. To quantify the density of biofilms formed under various conditions, biofilms were established on a Nunc TSP plate lid as described above. Pegs were rinsed in saline for $2 \mathrm{~min}$, then placed in a 96-well round-bottom Nunc plate with $150 \mu \mathrm{l}$ sterile saline and sonicated twice for $10 \mathrm{~min}$ in a Fisher FS 110 sonicator. Test wells were pooled and serially diluted for quantification by colony counts.

An alternative method was utilized to compare bacterial quantity by c.f.u. to quantification by metabolism via alamarBlue reduction. First, biofilms were established on a Nunc TSP 96-well plate lid as described above with $2.5 \times 10^{4}$ bacteria (PMN to bacteria ratio of $1: 0.01$ ). Pegs were rinsed in saline for $2 \mathrm{~min}$, then the lid was divided in half. Half the lid was treated with a combination of DNase and poly(L-aspartic acid) at concentrations of $66 \mu \mathrm{g}$ DNase $\mathrm{ml}^{-1}$ and $5 \mu \mathrm{M}$, respectively, for $20 \mathrm{~min}$. Following digestion, the contents of wells were removed, serially diluted in saline, and plated on LB agar. The treated and untreated lid halves were then placed into alamarBlue development medium (200 $\mu \mathrm{l}$ total well volume, comprising $130 \mu \mathrm{l}$ RPMI 1640x, $50 \mu \mathrm{l} 2 \times \mathrm{LB}$ broth and $20 \mu \mathrm{l}$ alamarBlue (Invitrogen) in a Costar 3603 well plate (Corning). Plates were read in a FLX 800 plate reader (BioTek instruments) every half hour for $24 \mathrm{~h}$ at $37{ }^{\circ} \mathrm{C}$ with shaking. Bacterial counts were derived from plotting the $\mathrm{V}_{50}$ of sample wells calculated by a Boltzmann sigmoidal non-linear regression against the $\mathrm{V}_{50}$ of standard curves generated by defined concentrations of bacteria that were plotted in a linear regression against log c.f.u.

Neutrophil lysis and induction of apoptosis to induce biofilm growth. To examine the role of available cellular constituents as well as living neutrophils in $P$. aeruginosa biofilm enhancement, we compared biofilm density in the presence of intact unstimulated neutrophils to that in the presence of lysed or apoptotic neutrophils. Lysed neutrophils were prepared the same way as living neutrophils, but were frozen and thawed $\left(-80{ }^{\circ} \mathrm{C}\right.$ for $\left.15 \mathrm{~min}\right)$ once prior to addition of bacteria. Apoptosis was induced by suspending neutrophils at $2.0 \times 10^{6}$ cells $\mathrm{ml}^{-1}$ in apoptosis buffer $(137 \mathrm{mM} \mathrm{NaCl}$, $2.7 \mathrm{mM} \mathrm{KCl}, 2 \mathrm{mM} \mathrm{MgCl} 2,5 \mathrm{mM}$ glucose, $10 \mathrm{mM}$ HEPES $\mathrm{pH} 7.4$, $0.05 \%$ delipidated BSA ) and UV irradiation $(254 \mathrm{~nm})$ on a Fotodyne 3-3000 UV transilluminator for $5 \mathrm{~min}$. Cells were then incubated at $37{ }^{\circ} \mathrm{C}$ with shaking (30 r.p.m.) in conical polypropylene tubes. Apoptosis was determined using an aliquot of $2 \times 10^{5}$ cells which were diluted and cytocentrifuged at 600 r.p.m. for $2 \mathrm{~min}$ on glass slides and stained with a modified Wright-Giemsa stain. Cells were counted as apoptotic if their nuclei lost segmentation and appeared round and darkly stained as described previously (Whitlock et al., 2000). At least 200 cells were counted per slide in a blinded fashion until $>80 \%$ apoptosis had been reached. Biofilms were grown for $24 \mathrm{~h}$ after the addition of bacteria, then assessed by CV staining as detailed above.

Light microscopy. Intact, lysed and apoptotic neutrophils were prepared as described above and P. aeruginosa strain PAO1 was added at a concentration ratio of 1:0.01 in an 18-well slide (total volume of $20 \mu \mathrm{l}$ ) (Ibidi). Slides were incubated at $37^{\circ} \mathrm{C}$ for $30 \mathrm{~min}$, then fixed with $1.3 \%$ glutaraldehyde $+33.3 \%$ ethanol at room temperature for 5 min. Fixative was removed and the slide was stained with a modified Wright-Giemsa stain. Slides were visualized on bright-field with an Axiovert 200M microscope (Carl Zeiss) using a $\times 63$ oil objective. Images were minimally level adjusted using Adobe Photoshop CS to show detail. 
Scanning electron microscopy (SEM). Biofilms of $P$. aeruginosa isolates $\left(2.5 \times 10^{4}\right.$ c.f.u. $)$ were grown in the presence or absence of neutrophils $\left(2.5 \times 10^{6}\right)(\mathrm{PMN}$ to bacteria ratio of $1: 0.01)$ for $48 \mathrm{~h}$. Pegs were rinsed briefly with $0.9 \%$ saline to remove non-biofilmassociated mass and were fixed with $2.5 \%$ glutaraldehyde in $0.1 \mathrm{M}$ cacodylate buffer $\left(\mathrm{pH} \mathrm{7.2)}\right.$ at $4{ }^{\circ} \mathrm{C}$ for $20 \mathrm{~h}$. The pegs were then washed in $0.1 \mathrm{M}$ cacodylate buffer for $10 \mathrm{~min}$, and rinsed in $\mathrm{ddH}_{2} \mathrm{O}$ briefly. The pegs were then dehydrated in $70 \%$ ethanol for $15 \mathrm{~min}$. Pegs were air-dried for a minimum of $72 \mathrm{~h}$, removed from the TSP reactor with a hot scalpel, and affixed to carbon stubs with epoxy resin and allowed to dry. Following gold sputter coating, SEM was performed using a JEOL 5800LV scanning electron microscope at low and high magnifications to visualize both gross as well as fine detail. Images were contrast/brightness-adjusted in Adobe Photoshop CS. All images of biofilms grown without neutrophils had a despeckle filter minimally applied to reduce background.

Verification of isogenic status of early and late CF $\boldsymbol{P}$. aeruginosa isolates. In order to confirm the lineage of the strains utilized in this report, a series of PCRs were run that amplify variable number tandem repeats (Onteniente et al., 2003). Briefly, this method amplifies tandem repeats of nucleotides of defined size that occur in variable number, which is related to lineage of the bacteria. Thus products of similar sizes indicate a closer lineage. Reaction conditions and primers were as described by Onteniente et al. (2003). Taq polymerase was Eppendorf MasterMix. Primers were purchased from IDT.

This assay resulted in seven of eight primers providing a product, and all of the reaction products of the early and late strains were identical (see Supplementary Fig. S1 in JMM Online). In all reactions, the CF early and late strains produced products of an identical size. Neither clinical strain produced a product from primer set 5915, indicating that this particular variable number tandem repeat was not present; however, a product was produced by PAO1 (Supplementary Fig. S1d). PAO1 produced differently sized products from the CF strain in all primer sets save 3496 . Coupled with previously published pulsefield data (Ogle et al., 1987), we are satisfied that these strains represent isogenic bacteria that have been isolated both at initial colonization (environmental) as well as after prolonged infection from a child with CF (host-adapted).
Statistical analysis. Data were analysed using Prism 4 (Graphpad version 4.03) or Excel (Microsoft Office 2003). Student's $t$-test was used to determine significance of paired data. One-way, and repeated measures ANOVA were performed on data where multiple testing resulted in datasets that required additional post-test analysis. Tukey's post test, where all datasets are compared to each other, and Dunnett's post test, where multiple datasets are compared to a single control set, were utilized as noted, with significance at a value no less than $P<0.05$.

\section{RESULTS AND DISCUSSION}

\section{$P$. aeruginosa biofilm density and quantity of associated bacteria are increased by the presence of human neutrophils}

Human neutrophils isolated from healthy volunteers were combined with $P$. aeruginosa strain PAO1 (Fig. 1a), an early $\mathrm{CF}$ isolate (Fig. 1b) and an isogenic late $\mathrm{CF}$ isolate (Fig. 1c) over a range of bacteria to neutrophil ratios spanning 4 logs. All ratios of bacteria to neutrophils tested resulted in a significant $(P<0.05)$ enhancement of biofilm density when compared to $P$. aeruginosa of equal concentration grown in the absence of neutrophils, as measured by CV staining. To verify that neutrophil-induced increases in biofilm density also result in an increase in the bacterial population, we examined the metabolism of the biofilm with an alamarBlue reduction viability assay, and compared the results to biofilm density as quantified by $\mathrm{CV}$ staining. A similar pattern of neutrophil-induced biofilm enhancement was found to exist between methods (Supplementary Fig. S2). Further, bacterial counts calculated by alamarBlue assay were determined to be similar to those when biofilms were digested and plated (data not shown). In sum, these results indicate that the presence of human neutrophils enhances early biofilm density and quantity of

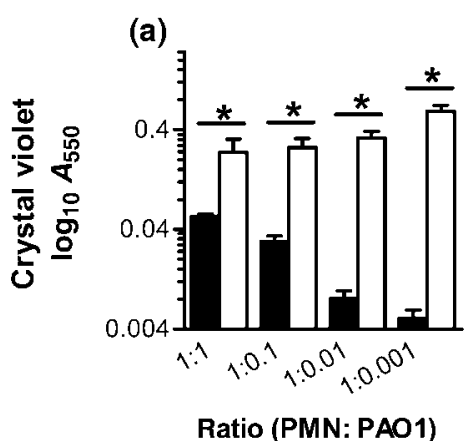

Ratio (PMN: PAO1)
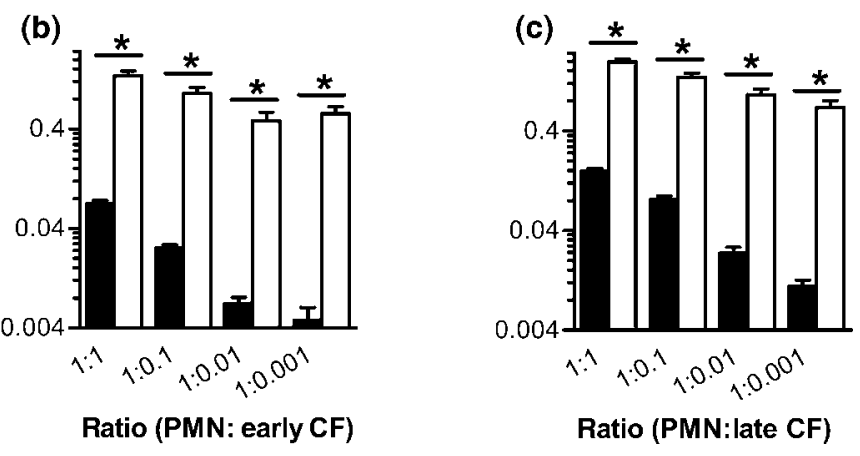

Fig. 1. P. aeruginosa biofilm formation is enhanced by human neutrophils. Biofilm density following $24 \mathrm{~h}$ of bacterial growth in the presence (open bars) or absence (black bars) of neutrophils. At time 0 , a fixed inoculum of neutrophils $\left(2.5 \times 10^{6}\right)$ was combined with $P$. aeruginosa at a $1: 1$ ratio, and decreasing 10-fold concentrations of bacteria to 1:0.001. Strains examined were PAO1 (a), a clinical CF strain isolated early after initial infection (b) and an isogenic mucoid strain isolated from the same patient 4 years later (c). Biofilm formation was quantified by CV staining, with correlated absorbance presented on a log ${ }_{10}$ scale. Significance of the addition of neutrophils $\left(^{*}\right)$ was calculated by Student's $t$-test at $P<0.05$, with comparisons as noted. The results represent the means of 10 independent experiments. 
bacteria. This effect was demonstrated with increases of $P$. aeruginosa biofilms from PAO1 as well as isogenic early and late CF isolates by orders of magnitude over that produced by the bacteria alone at $24 \mathrm{~h}$.

Though intrinsically capable of forming biofilms, in the absence of neutrophils, decreasing initial concentrations of $P$. aeruginosa (inocula ranging from $2.5 \times 10^{6}$ to $2.5 \times 10^{3}$ c.f.u.) resulted in a significant decrease in biofilm density from each concentration to the next, save the two most dilute samples (Fig. 1a-c). Under the conditions tested, a basal level of biofilm formation is reached at an inoculum of $2.5 \times 10^{4}$ c.f.u. and decreasing the bacterial concentration further has no effect in decreasing uninduced biofilm density. This is of particular interest, as the initial inoculum of $P$. aeruginosa entering the CF airway is likely quite small.

Neutrophils are short-lived cells, and in the presence of $P$. aeruginosa rapidly progress to death via necrosis or apoptosis. Necrotic cell death features loss of cell membrane integrity and the uncoordinated release of nuclear and cytoplasmic contents. Programmed cell death via apoptosis proceeds through a coordinated series of steps, which results in nuclear fragmentation and intense chromatin condensation, but maintenance of a near-tointact cytoplasmic membrane. We compared the level of biofilm induction resultant from combining bacteria with intact viable cells to that with lysed or apoptotic neutrophils. Within 30 min, many of the intact neutrophils had undergone lysis, and bacteria were seen to aggregate in the presence of necrotic debris (Fig. 2b). A similar effect was seen following $30 \mathrm{~min}$ of co-incubation with lysed neutrophils (Fig. 2c). In contrast, neutrophils induced to apoptosis prior to exposure to $P$. aeruginosa remained intact, and the bacteria remained dispersed (Fig. 2d). By $24 \mathrm{~h}$, viable and lysed neutrophils induced equivalent levels of biofilm enhancement, but apoptotic neutrophils induced significantly less when compared to the live PMNs $(P<0.001)$ (Fig. 2e). Together, these results demonstrate that apoptotic death of the neutrophil limits the availability of F-actin and DNA, which is required to enhance early $P$. aeruginosa biofilm formation. It is widely accepted that neutrophil apoptosis represents a beneficial response in reducing tissue injury from uncoordinated release of various cytotoxic components of the leukocyte. Our findings support the idea that in the presence of $P$. aeruginosa, neutrophil apoptosis also serves to limit the capacity of the pathogen to form a biofilm.

Biofilms of $P$. aeruginosa strain PAO1 (Fig. 3; Supplementary Fig. S3) as well as of the early and late CF strains (Supplementary Figs S4 and S5) were allowed to form in the presence of neutrophils, and compared by SEM to biofilms grown in the absence of neutrophils. The presence of neutrophils evoked clear differences. Neutrophil-induced biofilms largely covered each peg, while in the absence of neutrophils, only scattered aggregates of bacteria were visualized (Fig. 3; Supplementary Figs S3-S5). In addition,
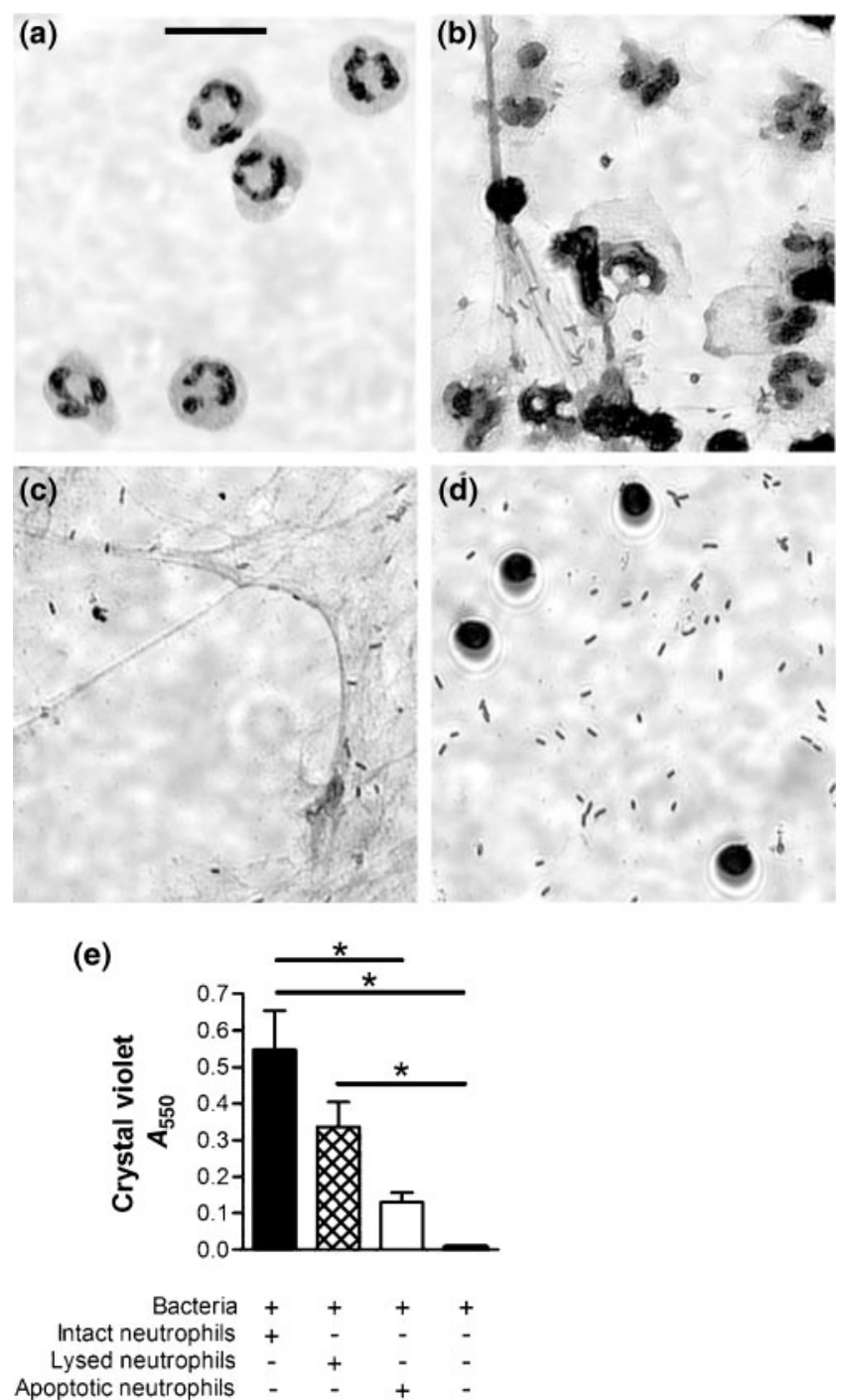

Fig. 2. $P$. aeruginosa responds differently to intact, lysed or apoptotic neutrophils. Intact and unstimulated neutrophils (a) were incubated with PAO1 (b), and compared to lysed (c) or apoptotic (d) neutrophils. Biofilms of PAO1 formed in the presence of these stages of neutrophil death show that intact neutrophils induce greatest biofilm enhancement, whereas apoptotic neutrophils do not enhance biofilm development (e). Microscopic examination (×63) of the localization of $P$. aeruginosa to these neutrophil types after a half-hour exposure shows that Pseudomonas preferentially associates with neutrophilic cellular products [necrotizing intact neutrophils (b) and neutrophil lysate (c)] contrasted to apoptotic neutrophils (d). Significance $\left(^{*}\right)$ was calculated by one-way ANOVA followed by Tukey's post-test at $P<0.001$. Photomicrographs and graph are representative of four independent experiments; bar, $10 \mu \mathrm{m}$.

the architecture of the biofilm was dramatically thicker and more developed in the presence of neutrophils. Generally, all three strains followed similar patterns in uninduced and neutrophil-induced biofilm formation at $48 \mathrm{~h}$. 

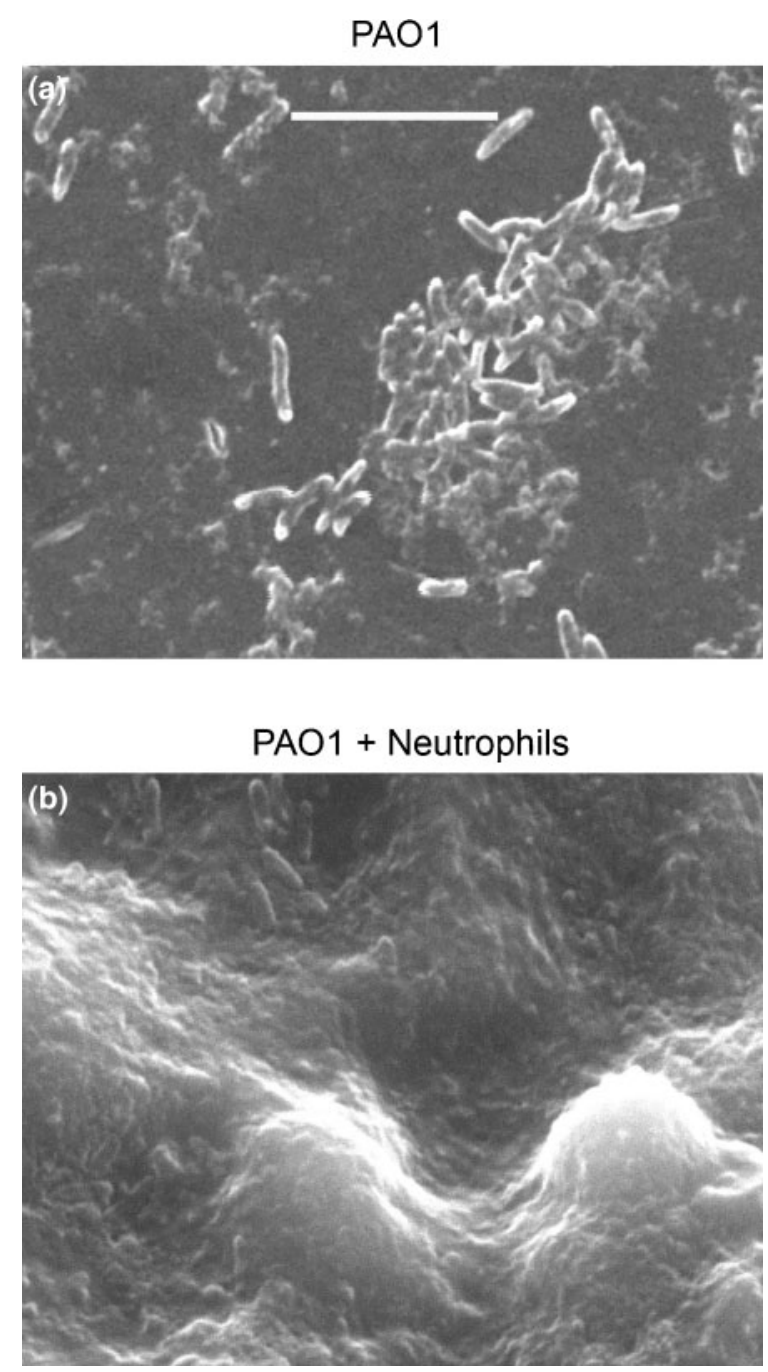

Fig. 3. SEM of neutrophil-induced enhancement of biofilm density and architecture. Biofilms of strain PAO1 were grown for $48 \mathrm{~h}$ in the presence or absence of human neutrophils. Examination by SEM at high magnification demonstrated that biofilms grown without human neutrophils were scattered sparsely on the reactor pegs (a), while biofilms grown with neutrophils (b) were much thicker and nearly confluent. Biofilms grown in the presence of human neutrophils have a more established architecture compared to biofilms grown in the absence of neutrophils. Bar, $8 \mu \mathrm{m}$. Photomicrographs are representative of three independent experiments.

\section{Disruption of neutrophil-induced $\boldsymbol{P}$. aeruginosa biofilms by anionic but not uncharged poly(amino acids)}

Previously, we have shown that polymers of F-actin and DNA are neutrophil products that enhance biofilm formation (Walker et al., 2005). Enhancement of P. aeruginosa biofilm development in the presence of neutrophils was lost in the presence of DNase, which enzymically depolymerizes DNA, and gelsolin, which severs F-actin into small fragments (Walker et al., 2005). Recently it has been reported that anionic poly(amino acids) can dissolve negatively charged F-actin and DNA bundles that are electrostatically associated via positively charged histones and other multivalent cations (Tang et al., 2005). Poly(aspartic acid) has a concentrated negative charge that has a twofold effect. Firstly, it sequesters the histone from the DNA and F-actin preventing it from serving as a charge linker between these two polymers (Tang et al., 2005). Secondly, its acidic nature causes depolymerization of F-actin to G-actin (Tang \& Janmey, 1996). We hypothesize that these actions could dismantle the early biofilm structure, as well as prevent initial formation or reformation after dissolution.

Peptides of equivalent length (median length of 74-mer) were compared for their capacity to disrupt a 24 -h-old biofilm of $P$. aeruginosa formed in the presence of human neutrophils. Homopeptide chains of anionic (Asp) ${ }_{74}$ and a polar but uncharged $(\mathrm{Ser})_{74}$ were analysed. When the $24 \mathrm{~h}$ biofilms were combined with these poly(amino acids) suspended in saline (free of neutrophil products not incorporated in the biofilm) for $3 \mathrm{~h}$, a significant concentration-related disruption of biofilms occurred in response to $(\text { Asp })_{74}$ (Fig. 4a). The effect of the anionic peptide is dose-related, as there was a significant decrease in biofilm density as the concentration of $(\text { Asp })_{74}$ was increased from 5 to $10 \mu \mathrm{M}$ (Fig. 4a). The polar but uncharged $(\mathrm{Ser})_{74}$ did not cause disruption of the biofilm at either concentration (Fig. 4a).

\section{Effect of poly(amino acid) chain length on biofilm disruption}

To establish whether or not the biofilm-disrupting effect of negatively charged peptides is related to the length of the polymer chain, we compared the effect of longer charged polypeptides. Using equimolar concentrations, amino acid chains of 260 residues were compared to the 74-mer polypeptides to determine the effect of chain length on biofilm disruption. Under these conditions, (Asp) 260 was more effective than (Asp) $)_{74}$ (Fig. 4b).

\section{Proteases reduce the capacity of charged poly(amino acids) to disrupt neutrophil-induced $P$. aeruginosa biofilms}

When poly(amino acids) were added directly to the milieu of necrotic neutrophils in which the biofilm was formed, the capacity of poly(amino acids) to disrupt biofilms was diminished (Fig. 5a) compared to reactions conducted in saline (Fig. 4). Under these conditions, neither (Asp) $)_{74}$ nor $(\mathrm{Ser})_{74}$ was able to disrupt biofilms. Further, a similar effect was observed when the long-chain and short-chain poly(aspartic acid) were compared (Fig. 5b). As both neutrophils and $P$. aeruginosa contain and/or synthesize an array of proteases, which degrade peptide chains, we tested the capacity of a protease inhibitor cocktail to restore the potential of poly(amino acids) to disrupt the biofilm under 
(a)

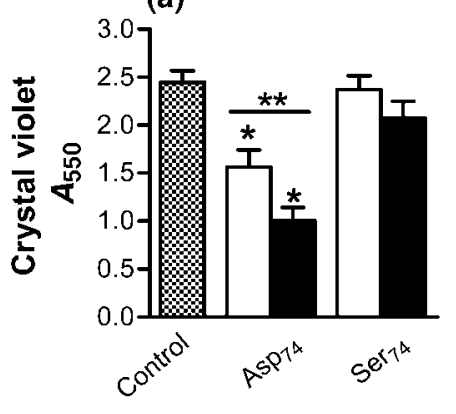

(b)

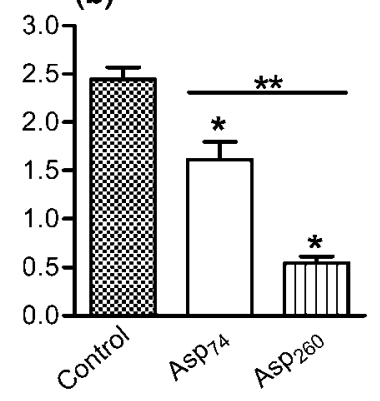

Fig. 4. $P$. aeruginosa biofilms induced by neutrophils are disrupted by electrostatically charged amino acid chains. (a) $P$. aeruginosa (strain PAO1) biofilms formed for $24 \mathrm{~h}$ in the presence of neutrophils were washed in saline and combined with poly(amino acids) of differing length and charge for $3 \mathrm{~h}$ at $37{ }^{\circ} \mathrm{C}$. (Asp) $)_{74}$ at $5 \mu \mathrm{M}$ (open bars) exhibited disruption of the biofilm; this effect was significantly greater at $10 \mu \mathrm{M}$ (black bars). The uncharged (Ser) ${ }_{74}$ had no effect. (b) Using poly(amino acids) at a concentration of $5 \mu \mathrm{M}$, the longer (Asp) 260 was more effective than the shorter $(A s p)_{74}$. All assays are as measured by absorbance from CV staining. Significance $\left(^{*}\right)$ was calculated by one-way ANOVA followed by Dunnett's post-test at $P<0.05$ and $\left.{ }^{\star \star}\right)$ two-tailed $t$-test $P<0.05$. The results represent the means of four independent experiments.

these conditions. In the presence of protease inhibitors, $(\text { Asp })_{260}$ significantly disrupted the biofilm compared to the untreated control (Fig. 5c). With a sevenfold greater concentration of protease inhibitors, there exists an even greater restoration of the ability of $(\mathrm{Asp})_{260}$ to disrupt biofilms, the reduction in biofilm density increasing from $25 \%$ to $52 \%$ (Fig. 5 c). When the biofilms were removed from the overnight culture material containing the products of necrotic neutrophils (such as proteases of bacterial and neutrophil origin), and washed once in saline, addition of (Asp) ${ }_{260}$ exhibited a significant disruptive effect, with no additional enhancement achieved by the addition of the protease inhibitor cocktail (Fig. 5d). These data support the conclusion that an anionic polypeptide can disrupt $P$. aeruginosa biofilms formed in the presence of human neutrophils, but proteolytic degradation, likely mediated by both bacteria and neutrophils, is a significant, though potentially solvable, obstacle to the use of poly(amino acids) in a neutrophil-rich environment.

Proteases in the lung environment are known to destroy a number of host defence mechanisms (Elkington et al., 2005; Roghanian et al., 2006), and the same seems to apply to poly(amino acids) in this system. A wide variety of modifications are now known that protect peptides from proteases, such as using $\mathrm{D}$ isomers or interspersing aspartic acid residues with non-naturally occurring amino acids. Studies by our group are under way to both protect the poly(aspartic acid) chain as well as to identify negatively charged polymers with greater intrinsic resistance to proteolytic degradation.

\section{Ciprofloxacin and tobramycin are incapable of disrupting neutrophil-induced biofilms}

Currently, the standard treatment for $P$. aeruginosa is antibiotics such as ciprofloxacin or tobramycin, although treatment with DNase has also been associated with decreased $P$. aeruginosa infectious load in the CF airway (Frederiksen et al., 2006; Gibson et al., 2003). In order to compare the relative effectiveness of polypeptides in dispersing $P$. aeruginosa biofilm in this system, we tested the ability of clinically relevant antibiotics to disrupt a biofilm induced by human neutrophils. P. aeruginosa biofilms (24-h-old) were exposed to twofold dilutions of either ciprofloxacin (Fig. 6a) or tobramycin (Fig. 6b) in clinically relevant concentrations ranging from $8 \mu \mathrm{g}$ antibiotic $\mathrm{ml}^{-1}$ to $1024 \mu \mathrm{g}$ antibiotic $\mathrm{ml}^{-1}$. The biofilm MIC was determined to be $128 \mu \mathrm{g}$ antibiotic $\mathrm{ml}^{-1}$ and $256 \mu \mathrm{g}$ antibiotic $\mathrm{ml}^{-1}$ for ciprofloxacin and tobramycin, respectively, under these conditions (data not shown). The biofilms were exposed for $3 \mathrm{~h}$ to antibiotics then quantified as described above with CV. Even under the highest concentration, the antibiotics tested demonstrated no capacity to disrupt the neutrophil-induced $P$. aeruginosa biofilm (Fig. 6), consistent with the well-established principle that conventional antibiotics are relatively less effective against biofilms (Costerton et al., 1987; Hoyle \& Costerton, 1991; Prosser et al., 1987). Azithromycin, an antibiotic ineffective against planktonic $P$. aeruginosa, has been shown to have an antimicrobial effect against $P$. aeruginosa biofilms (Moskowitz et al., 2004), to retard biofilm development (Gillis \& Iglewski, 2004), and to block quorum sensing and alginate polymer formation (Hoffmann et al., 2007). While azithromycin has not been shown to disrupt an established $P$. aeruginosa biofilm in vitro (Gillis \& Iglewski, 2004), additional studies are warranted to fully define the anti-biofilm properties of this agent.

\section{Anionic poly(amino acid) chains have the capacity to prevent $\boldsymbol{P}$. aeruginosa biofilm formation}

Although treatment of established $P$. aeruginosa biofilms likely represents the most relevant clinical scenario, in certain situations, biofilm formation is a highly predictable event. If agents existed that were effective in preventing the formation of the biofilm, strategies could be employed to reduce the potential for biofilm formation. We tested the capacity of $(\mathrm{Asp})_{74}$ or $(\mathrm{Ser})_{74}$ to inhibit biofilm development when added directly to a suspension of $P$. aeruginosa and neutrophils (Fig. 7). Biofilm development was quantified after $24 \mathrm{~h}$ incubation as detailed above. Uncharged $(\mathrm{Ser})_{74}$ demonstrated no capacity to prevent biofilm formation, while $(\text { Asp })_{74}$ was significantly effective at preventing the formation of biofilms.

The robust activity of this amino acid chain, $(\mathrm{Asp})_{74}$, in the prevention of biofilm formation led to the question of an alternative mechanism of disruption in this system. To address this, the toxicity or growth-enhancing effect of all the components used in the peptide assays was tested individually with $P$. aeruginosa and, when appropriate, in 
(a)

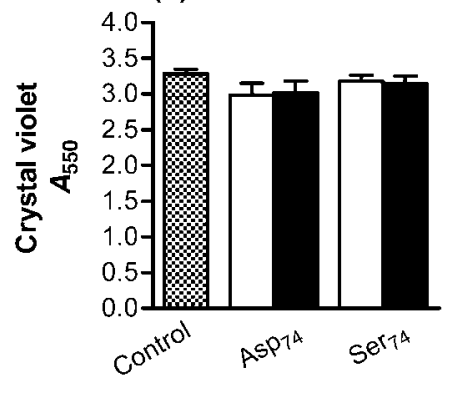

(c)

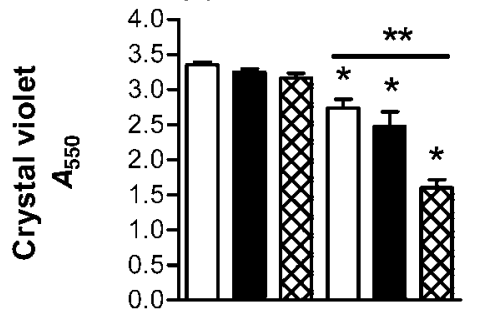

Protease inhibitor $-1 \times 7 \times-1 \times 7 \times$ (b)

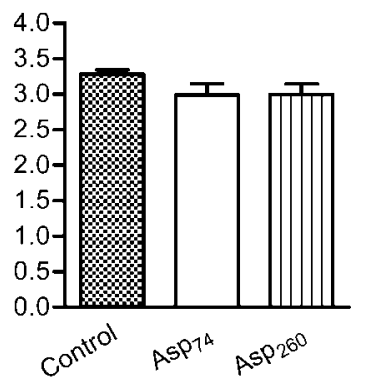

(d)

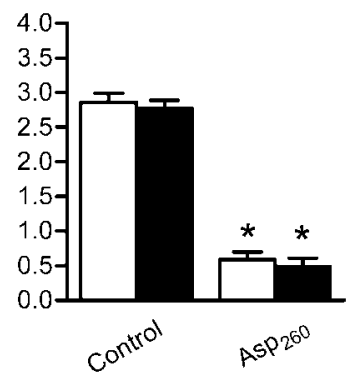

Fig. 5. Proteolytic degradation reduces the capacity of charged poly(amino acids) to disrupt neutrophil-induced $P$. aeruginosa biofilms. (a) When poly(amino acids) of differing concentration were combined with a neutrophil-induced $24 \mathrm{~h} P$. aeruginosa biofilm in the presence of overnight culture material (OCM), there was no significant effect on biofilm disruption at $5 \mu \mathrm{M}$ (open bars) or $10 \mu \mathrm{m}$ (black bars). (b) Under identical conditions, when the capacity of (Asp) ${ }_{74}$ and (Asp) 260 to disrupt biofilms was tested there was not a significant effect in the presence of OCM. (c) When (Asp) 260 (open bars) was combined with a protease inhibitor cocktail (black bars) in the presence of the OCM, a significant disruption of the biofilm was achieved, which was further increased when the protease inhibitor concentration was increased to sevenfold (crosshatched bars). (d) When the biofilms were removed from the OCM and washed once in saline, $(\text { Asp })_{260}$ exhibited a significant disruptive effect (open bar). No enhancement was achieved by the addition of a protease inhibitor cocktail (black bars) following removal of the neutrophil cellular milieu. All assays are as measured by absorbance from CV staining. Significance $\left(^{*}\right)$ was calculated by one-way ANOVA followed by Dunnett's post-test at $P<0.05$ and $\left.{ }^{* \star}\right)$ two-tailed $t$-test $P<0.05$. The results represent the means of three independent experiments.

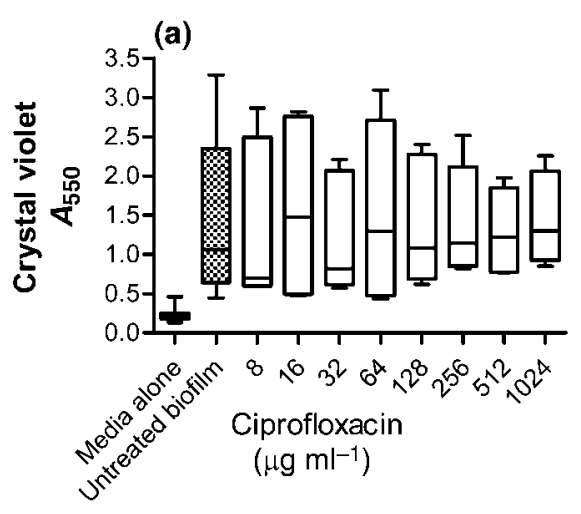

(b)

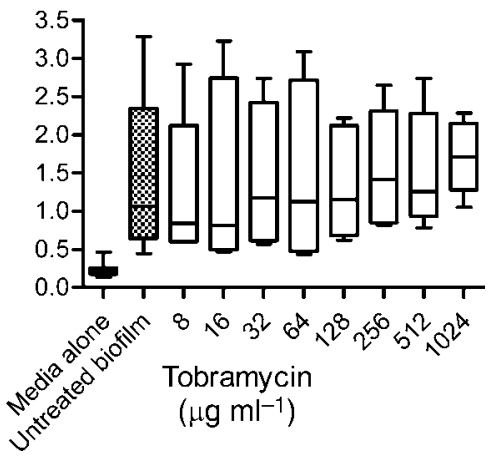

Fig. 6. Neutrophil-induced $P$. aeruginosa biofilms are not disrupted by anti-pseudomonal antibiotics. Neutrophil-induced PAO1 biofilms formed at a 1:0.01 ratio of bacteria to neutrophils were exposed to the antibiotics ciprofloxacin (a) or tobramycin (b) for $3 \mathrm{~h}$ at $37{ }^{\circ} \mathrm{C}$ at concentrations ranging from 8 to $1024 \mu \mathrm{g} \mathrm{ml} l^{-1}$. The biofilm density was then quantified by $\mathrm{CV}$ staining. No disruption of the biofilm was observed in response to either antibiotic over the range of dilutions tested. An untreated biofilm, as well as a media-only control, were included to serve as a reference of biofilm formation and background staining. Both assays are as measured by absorbance from CV staining. No significant differences were observed as calculated by the Kruskal-Wallis test followed by Dunn's multiple comparison test to the untreated biofilm at $P<0.05$. The results represent the means of three independent experiments. 


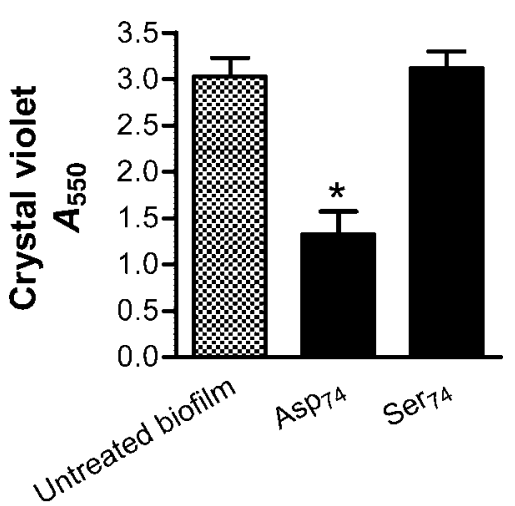

Fig. 7. Negative poly(amino acid) chains possess capacity to prevent neutrophil-induced enhancement of $P$. aeruginosa biofilm formation. Short-chain (Asp) 74 and (Ser) 74 at $10 \mu \mathrm{M}$ were added at time 0 to $P$. aeruginosa PAO1 combined with neutrophils (1:0.01 ratio). Following $24 \mathrm{~h}$ incubation at $37^{\circ} \mathrm{C}$, anionic $(\text { Asp })_{74}$ prevented biofilm formation while neutral $(\mathrm{Ser})_{74}$ displayed no effect. Assays are as measured by absorbance from CV staining. Significance $\left(^{*}\right)$ was calculated by one-way ANOVA followed by Dunnett's post-test at $P<0.05$ and two-tailed $t$-test $P<0.05$. The results represent the mean of three independent experiments.

combination. We found that within assays, the amount of bacteria recovered from pooled wells was consistent, and that neither $(\mathrm{Asp})_{74}$ nor $(\mathrm{Ser})_{74}$ displayed enhanced growth or bactericidal properties (not shown). In addition, the protease inhibitors pepstatin A, leupeptin and aprotinin alone or in combination displayed no antimicrobial effect.

\section{DNase disrupts $\boldsymbol{P}$. aeruginosa biofilms, and prevents their formation in the presence of neutrophils}

Previously, our group and others have shown the capacity of DNase to disrupt $P$. aeruginosa biofilms (Frederiksen et al., 2006; Walker et al., 2005; Whitchurch et al., 2002), supporting the concept that DNA is also an essential component of the 'scaffolding'. Under the conditions used in Fig. 4, exposure to DNase for $3 \mathrm{~h}$ disrupts 24-h-old neutrophil-induced biofilms (Fig. 8a). Likewise, DNase was able to significantly prevent biofilm formation in the presence of neutrophils (Fig. 8b), under the conditions described for Fig. 7. In contrast, RNase demonstrated no capacity to disrupt (Fig. 8a) or prevent (Fig. 8b) biofilm formation.

\section{Poly(aspartic acid) works in conjunction with DNase to disrupt biofilms}

While concentrated DNase (Fig. 8a) or poly(aspartic acid) (Fig. 4) are both capable of disrupting an early biofilm over the course of a $3 \mathrm{~h}$ exposure, during DNase therapy in the CF airway it is likely that biofilms are exposed to DNase over a wide range of concentrations, and the effective (a)

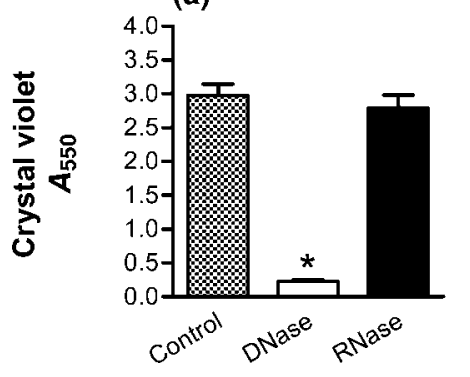

(b)

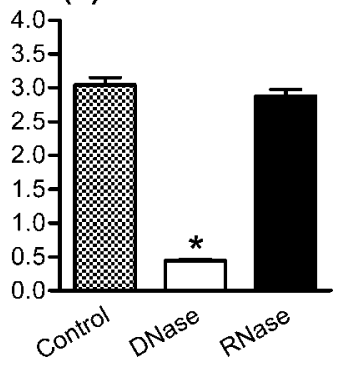

Fig. 8. Neutrophil-induced $P$. aeruginosa biofilms are both disrupted and prevented by DNase. (a) $P$. aeruginosa strain PAO1 biofilms were grown for $24 \mathrm{~h}$ in the presence of neutrophils ( $1: 0.01$ ratio). The biofilms were then exposed to either DNase (open bars) or RNase (black bars) for $3 \mathrm{~h}$. The neutrophil-induced biofilm was significantly disrupted by DNase, but not by RNase. (b) DNase or RNase was combined with $P$. aeruginosa strain PAO1 and neutrophils ( $1: 0.01$ ratio), and a biofilm was allowed to form for $24 \mathrm{~h}$. The presence of DNase prevented the formation of the biofilm, while RNase had no effect. Assays are as measured by absorbance from CV staining. Significance $\left({ }^{*}\right)$ was calculated by one-way ANOVA followed by Dunnett's post-test at $P<0.05$. The results represent the means of three independent experiments.

duration of exposure may be quite brief. Furthermore, in the setting of chronic infections, biofilms are likely more than $24 \mathrm{~h}$ old. We tested the capacity of DNase and $(\text { Asp })_{260}$ to disrupt biofilms when administered over a shorter period, at lower concentrations, and with thicker and more mature biofilms.

When the duration of exposure to DNase was reduced to only $10 \mathrm{~min}$, a 24 -h-old neutrophil-induced biofilm was still reduced by $75 \%$ (Fig. 9a). However, a biofilm grown for $48 \mathrm{~h}$ was reduced by only $42 \%$ by $10 \mathrm{~min}$ of DNase treatment (Fig. 9b). Likewise, biofilms at later stages of growth $(48 \mathrm{~h})$ also had greater resistance to disruption with (Asp) ${ }_{260}$ and DNase; however, in combination these agents were effective in disrupting biofilms $(P<0.01)$. Exposing a $24 \mathrm{~h}$ neutrophil-induced biofilm for only 10 min to (Asp) 260 resulted in a $49 \%$ reduction in biofilm density (Fig. 9a). When DNase was used in combination with (Asp) 260 , the mixture resulted in an $82 \%$ reduction (Fig. 9a). (Asp) ${ }_{260}$ alone could not reduce the density of a more mature $48 \mathrm{~h}$ biofilm, but when used in conjunction with DNase, (Asp) 260 was able to reduce the biofilm by $78 \%$ (Fig. 8b).

The combined effect of $(\text { Asp })_{260}$ with DNase was dosedependent (Fig. 9c). By decreasing DNase concentration fourfold $\left(8.25 \mu \mathrm{g}\right.$ DNase $\left.\mathrm{ml}^{-1}\right)$, only a $16 \%$ decrease in the biofilm density of a $48 \mathrm{~h}$ biofilm was achieved after $10 \mathrm{~min}$ treatment (Fig. 9c), compared to $42 \%$ at the $33 \mu \mathrm{g}$ DNase $\mathrm{ml}^{-1}$ concentration (Fig. 9b). When the lower concentration of DNase was used in combination with $(\mathrm{Asp})_{260}$, the density of the biofilm was reduced by $67 \%$ (Fig. 9c), demonstrating a strong synergistic effect between the 

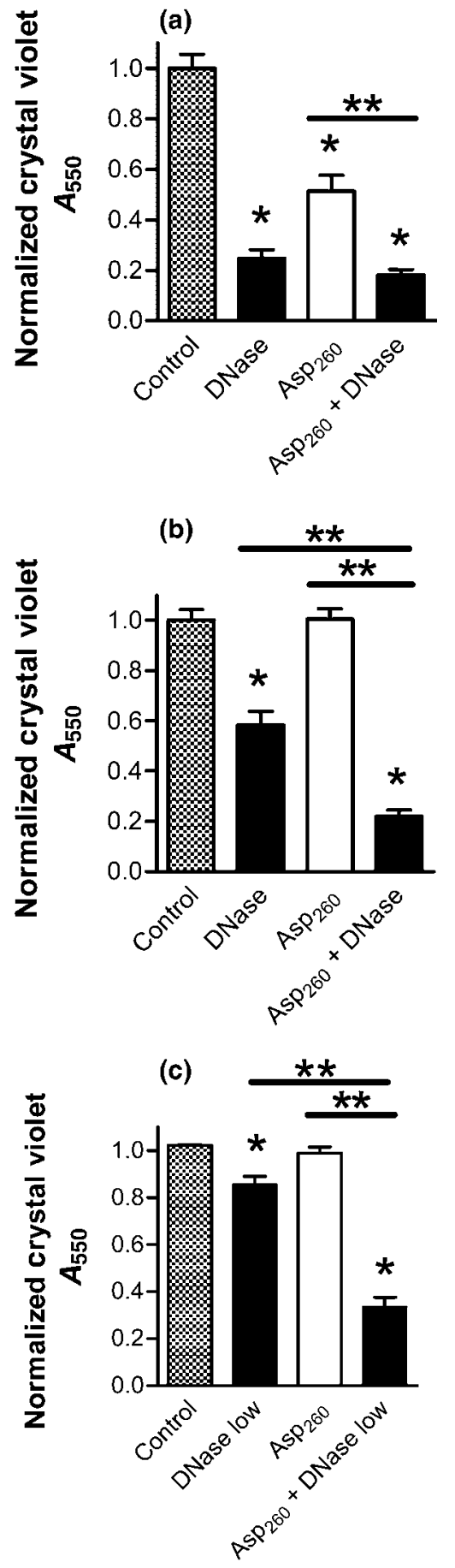

enzyme and poly(aspartic acid). Thus, under conditions in which DNase as a single agent has little effect, the presence of (Asp) 260 significantly enhances the capacity of DNase to disrupt a biofilm formed in the presence of neutrophils.

Parallel experiments were conducted with the early and late CF strains. Neutrophil-induced biofilms of the early CF strain (24 h of growth) were not disrupted by a $10 \mathrm{~min}$ exposure to DNase (Supplementary Fig. S6a), while the late CF strain demonstrated a modest disruption (Supplementary Fig. S6c). However, for both strains, the effect
Fig. 9. Poly(aspartic acid) works in cooperation with DNase to disrupt neutrophil-induced $P$. aeruginosa biofilms. PAO1 biofilms were allowed to form for 24 or $48 \mathrm{~h}$ in the presence of neutrophils at a 1:0.01 ratio of bacteria to neutrophils. The biofilms were then washed in saline and exposed to either poly(aspartic acid) alone (open bars) or combined with DNase (black bars). In all experiments, exposure to DNase and/or poly(aspartic acid) was for $10 \mathrm{~min}$ at $37{ }^{\circ} \mathrm{C}$ in saline. (a) As single agents, (Asp) $260(5 \mu \mathrm{M})$ and $33 \mu \mathrm{g}$ DNase $\mathrm{ml}^{-1}$ effectively disrupted 24 -h-old biofilms. (b) Using a $48 \mathrm{~h}$ biofilm, $33 \mu \mathrm{g}$ DNase $\mathrm{ml}^{-1}$, but not (Asp) $260(5 \mu \mathrm{M})$, was effective as a single agent. However, a significant increase in biofilm disruption was achieved when (Asp) ${ }_{260}$ was combined with DNase. (c) Using a $48 \mathrm{~h}$ biofilm and a lower concentration of $8.25 \mu \mathrm{g}$ DNase $\mathrm{ml}^{-1}$, a synergistic effect of $(\text { Asp })_{260}(5 \mu \mathrm{M})$ and DNase was achieved. Assay is as measured by absorbance from CV staining. Significance $\left(^{*}\right)$ was calculated by one-way ANOVA followed by Dunnett's post-test at $P<0.05$ and $\left({ }^{\star \star}\right)$ two-tailed $t$ test $P<0.05$. The results represent the means of three independent experiments.

of DNase was significantly increased by the addition of $(\text { Asp })_{260}$ (Supplementary Fig. S6a, c). The synergistic effect of DNase and (Asp) 260 was also apparent following $48 \mathrm{~h}$ of biofilm growth for both the early and late CF strains (Supplementary Fig. S6b, d). Perhaps of greatest clinical interest were attempts to disrupt the mucoid late CF strain that had been allowed to form for $48 \mathrm{~h}$ in the presence of neutrophils (Supplementary Fig. S6d). Under these conditions, only the combination of DNase and (Asp) $)_{260}$ achieved significant disruption of the biofilm (Supplementary Fig. S6d).

The markedly decreased DNase effect in the context of the treatment of thicker $48 \mathrm{~h}$ biofilms, or with a mucoid strain of $P$. aeruginosa recovered later in CF infection, likely reflects an increased polysaccharide concentration that may obscure DNA cleavage sites. However, the synergistic effect of DNase with poly(aspartic acid) may stem from the capacity of poly(aspartic acid) to sequester histones as well as disassociating F-actin along with associated bacteria and saccharides, allowing DNase exposure to a greater number of sites for cleavage in the actin-DNA polymer.

The CF airway is particularly vulnerable to $P$. aeruginosa infection, where these bacteria are able to survive indefinitely in the presence of an exuberant recruitment of neutrophils. Necrosis of the short-lived neutrophil occurs consistently and rapidly, while clearance of the cellular debris is ineffective, resulting in the enrichment of already viscous mucus with both DNA and F-actin (Lethem et al., 1990; Rubin, 2007; Sheils et al., 1996). Additional DNA of bacterial origin is present in the CF airway as well (Lethem et al., 1990). The production of a $P$. aeruginosa biofilm is based on the association of the bacteria with a matrix, either self-produced or exogenously provided (Costerton et al., 1999; Hentzer et al., 2001; Sriramulu et al., 2005). In humans, focal infection by $P$. aeruginosa, and subsequent biofilm formation, always 
occurs in association with an exuberant inflammatory response. $P$. aeruginosa infection of wounds, burns or the cornea of contact lens wearers occurs only after neutrophils have accumulated at the site of injury. In the case of the CF airway, the released chromosomal DNA with associated histones and F-actin provide a ready framework for Pseudomonas to occupy and proliferate. Strands of negatively charged DNA polymerize with negatively charged F-actin filaments by interaction with positively charged species such as histones and cations. P. aeruginosa associates in turn with this structure by binding either DNA or F-actin (van Schaik et al., 2005; Walker et al., 2005).

The presence of a framework of neutrophil DNA and F-actin may help to explain the efficacy of inhaled DNase in $\mathrm{CF}$, which is associated with a decrease in infectious burden or frequency of pulmonary exacerbations (Frederiksen et al., 2006; Robinson, 2002). While improved sputum clearance is generally beneficial in controlling CF airway infection, the potential of DNase may be more fully realized by the addition of anionic polymers to disrupt biofilms in vivo. We have demonstrated that the presence of abundant F-actin and DNA in a neutrophil-rich environment is exploited by $P$. aeruginosa to ensure survival via biofilm development. Thus accounting for the incorporation of neutrophil products into the medically relevant $P$. aeruginosa biofilm is a logical advancement in our understanding of this extraordinary pathogen. It is conceivable that strategies to disrupt F-actin, as an adjunct to treatment with DNase and antibiotics, will represent an incremental improvement in our approach to treating chronic $P$. aeruginosa infection in the CF airway.

\section{ACKNOWLEDGEMENTS}

This work was supported by the following grants and foundations: HL090991 and HL34303 from the National Institutes of Health, a Cystic Fibrosis Foundation Research Grant (NICK07G0), The Rebecca Runyon Bryan Chair in Cystic Fibrosis, Colorado Bioscience Discovery Evaluation Grant and The Max and Yetta Karasik Foundation.

\section{REFERENCES}

Balfour-Lynn, I. M. (1999). The protease-antiprotease battle in the cystic fibrosis lung. J R Soc Med 92 (Suppl. 37), 23-30.

Baumgartner, A. \& Grand, M. (2006). Bacteriological quality of drinking water from dispensers (coolers) and possible control measures. J Food Prot 69, 3043-3046.

Burns, J. L., Gibson, R. L., McNamara, S., Yim, D., Emerson, J., Rosenfeld, M., Hiatt, P., McCoy, K., Castile, R. \& other authors (2001). Longitudinal assessment of Pseudomonas aeruginosa in young children with cystic fibrosis. J Infect Dis 183, 444-452.

Costerton, J. W., Cheng, K. J., Geesey, G. G., Ladd, T. I., Nickel, J. C., Dasgupta, M. \& Marrie, T. J. (1987). Bacterial biofilms in nature and disease. Annu Rev Microbiol 41, 435-464.

Costerton, J. W., Stewart, P. S. \& Greenberg, E. P. (1999). Bacterial biofilms: a common cause of persistent infections. Science $\mathbf{2 8 4}$ $1318-1322$.
Drenkard, E. \& Ausubel, F. M. (2002). Pseudomonas biofilm formation and antibiotic resistance are linked to phenotypic variation. Nature 416, 740-743.

Elkington, P. T., O’Kane, C. M. \& Friedland, J. S. (2005). The paradox of matrix metalloproteinases in infectious disease. Clin Exp Immunol $142,12-20$.

Emerson, J., Rosenfeld, M., McNamara, S., Ramsey, B. \& Gibson, R. L. (2002). Pseudomonas aeruginosa and other predictors of mortality and morbidity in young children with cystic fibrosis. Pediatr Pulmonol 34, 91-100.

Ernst, R. K., Yi, E. C., Guo, L., Lim, K. B., Burns, J. L., Hackett, M. \& Miller, S. I. (1999). Specific lipopolysaccharide found in cystic fibrosis airway Pseudomonas aeruginosa. Science 286, 1561-1565.

Frederiksen, B., Pressler, T., Hansen, A., Koch, C. \& Hoiby, N. (2006). Effect of aerosolized rhDNase (Pulmozyme) on pulmonary colonization in patients with cystic fibrosis. Acta Paediatr 95, 1070-1074.

Gibson, R. L., Burns, J. L. \& Ramsey, B. W. (2003). Pathophysiology and management of pulmonary infections in cystic fibrosis. $A m \mathrm{~J}$ Respir Crit Care Med 168, 918-951.

Gillis, R. J. \& Iglewski, B. H. (2004). Azithromycin retards Pseudomonas aeruginosa biofilm formation. J Clin Microbiol 42, 5842-5845.

Haslett, C., Guthrie, L. A., Kopaniak, M. M., Johnston, R. B., Jr \& Henson, P. M. (1985). Modulation of multiple neutrophil functions by preparative methods or trace concentrations of bacterial lipopolysaccharide. Am J Pathol 119, 101-110.

Hentzer, M., Teitzel, G. M., Balzer, G. J., Heydorn, A., Molin, S., Givskov, M. \& Parsek, M. R. (2001). Alginate overproduction affects Pseudomonas aeruginosa biofilm structure and function. J Bacteriol 183, 5395-5401.

Hoffmann, N., Lee, B., Hentzer, M., Rasmussen, T. B., Song, Z., Johansen, H. K., Givskov, M. \& Hoiby, N. (2007). Azithromycin blocks quorum sensing and alginate polymer formation and increases the sensitivity to serum and stationary-growth-phase killing of Pseudomonas aeruginosa and attenuates chronic $P$. aeruginosa lung infection in $\mathrm{Cftr}^{-1-}$ mice. Antimicrob Agents Chemother 51, 3677-3687.

Hoyle, B. D. \& Costerton, J. W. (1991). Bacterial resistance to antibiotics: the role of biofilms. Prog Drug Res 37, 91-105.

Khan, T. Z., Wagener, J. S., Bost, T., Martinez, J., Accurso, F. J. \& Riches, D. W. (1995). Early pulmonary inflammation in infants with cystic fibrosis. Am J Respir Crit Care Med 151, 1075-1082.

Khan, N. H., Ishii, Y., Kimata-Kino, N., Esaki, H., Nishino, T., Nishimura, M. \& Kogure, K. (2007). Isolation of Pseudomonas aeruginosa from open ocean and comparison with freshwater, clinical, and animal isolates. Microb Ecol 53, 173-186.

Klausen, M., Heydorn, A., Ragas, P., Lambertsen, L., AaesJorgensen, A., Molin, S. \& Tolker-Nielsen, T. (2003). Biofilm formation by Pseudomonas aeruginosa wild type, flagella and type IV pili mutants. Mol Microbiol 48, 1511-1524.

Lethem, M. I., James, S. L., Marriott, C. \& Burke, J. F. (1990). The origin of DNA associated with mucus glycoproteins in cystic fibrosis sputum. Eur Respir J 3, 19-23.

Moskowitz, S. M., Foster, J. M., Emerson, J. \& Burns, J. L. (2004). Clinically feasible biofilm susceptibility assay for isolates of Pseudomonas aeruginosa from patients with cystic fibrosis. J Clin Microbiol 42, 1915-1922.

Ogle, J. W., Janda, J. M., Woods, D. E. \& Vasil, M. L. (1987). Characterization and use of a DNA probe as an epidemiological marker for Pseudomonas aeruginosa. J Infect Dis 155, 119-126.

Onteniente, L., Brisse, S., Tassios, P. T. \& Vergnaud, G. (2003). Evaluation of the polymorphisms associated with tandem repeats for Pseudomonas aeruginosa strain typing. J Clin Microbiol 41, 4991-4997. 
Parad, R. B., Gerard, C. J., Zurakowski, D., Nichols, D. P. \& Pier, G. B. (1999). Pulmonary outcome in cystic fibrosis is influenced primarily by mucoid Pseudomonas aeruginosa infection and immune status and only modestly by genotype. Infect Immun 67, 4744-4750.

Perks, B. \& Shute, J. K. (2000). DNA and actin bind and inhibit interleukin-8 function in cystic fibrosis sputa: in vitro effects of mucolytics. Am J Respir Crit Care Med 162, 1767-1772.

Prosser, B. L., Taylor, D., Dix, B. A. \& Cleeland, R. (1987). Method of evaluating effects of antibiotics on bacterial biofilm. Antimicrob Agents Chemother 31, 1502-1506.

Robinson, P. J. (2002). Dornase alfa in early cystic fibrosis lung disease. Pediatr Pulmonol 34, 237-241.

Roghanian, A., Drost, E. M., MacNee, W., Howie, S. E. \& Sallenave, J. M. (2006). Inflammatory lung secretions inhibit dendritic cell maturation and function via neutrophil elastase. Am J Respir Crit Care Med 174, 1189-1198.

Roum, J. H., Buhl, R., McElvaney, N. G., Borok, Z. \& Crystal, R. G. (1993). Systemic deficiency of glutathione in cystic fibrosis. J Appl Physiol 75, 2419-2424.

Rubin, B. K. (2007). Mucus structure and properties in cystic fibrosis. Paediatr Respir Rev 8, 4-7.

Schaedel, C., de Monestrol, I., Hjelte, L., Johannesson, M., Kornfalt, R., Lindblad, A., Strandvik, B., Wahlgren, L. \& Holmberg, L. (2002). Predictors of deterioration of lung function in cystic fibrosis. Pediatr Pulmonol 33, 483-491.

Sheils, C. A., Kas, J., Travassos, W., Allen, P. G., Janmey, P. A., Wohl, M. E. \& Stossel, T. P. (1996). Actin filaments mediate DNA fiber formation in chronic inflammatory airway disease. Am J Pathol 148, 919-927.

Singh, P. K., Schaefer, A. L., Parsek, M. R., Moninger, T. O., Welsh, M. J. \& Greenberg, E. P. (2000). Quorum-sensing signals indicate that cystic fibrosis lungs are infected with bacterial biofilms. Nature 407, 762-764.

Spencer, D. H., Kas, A., Smith, E. E., Raymond, C. K., Sims, E. H., Hastings, M., Burns, J. L., Kaul, R. \& Olson, M. V. (2003). Wholegenome sequence variation among multiple isolates of Pseudomonas aeruginosa. J Bacteriol 185, 1316-1325.

Sriramulu, D. D., Lünsdorf, H., Lam, J. S. \& Römling, U. (2005). Microcolony formation: a novel biofilm model of Pseudomonas aeruginosa for the cystic fibrosis lung. J Med Microbiol 54, 667-676.

Tang, J. X. \& Janmey, P. A. (1996). The polyelectrolyte nature of F-actin and the mechanism of actin bundle formation. J Biol Chem 271, 8556-8563.

Tang, J. X., Wen, Q., Bennett, A., Kim, B., Sheils, C. A., Bucki, R. \& Janmey, P. A. (2005). Anionic poly(amino acid)s dissolve F-actin and DNA bundles, enhance DNase activity, and reduce the viscosity of cystic fibrosis sputum. Am J Physiol Lung Cell Mol Physiol 289, L599L605.

van Schaik, E. J., Giltner, C. L., Audette, G. F., Keizer, D. W., Bautista, D. L., Slupsky, C. M., Sykes, B. D. \& Irvin, R. T. (2005). DNA binding: a novel function of Pseudomonas aeruginosa type IV pili. J Bacteriol 187, 1455-1464.

Walker, T. S., Tomlin, K. L., Worthen, G. S., Poch, K. R., Lieber, J. G., Saavedra, M. T., Fessler, M. B., Malcolm, K. C., Vasil, M. L. \& Nick, J. A. (2005). Enhanced Pseudomonas aeruginosa biofilm development mediated by human neutrophils. Infect Immun 73, 3693-3701.

Whitchurch, C. B., Tolker-Nielsen, T., Ragas, P. C. \& Mattick, J. S. (2002). Extracellular DNA required for bacterial biofilm formation. Science 295, 1487.

Whitlock, B. B., Gardai, S., Fadok, V., Bratton, D. \& Henson, P. M. (2000). Differential roles for $\alpha_{M} \beta_{2}$ integrin clustering or activation in the control of apoptosis via regulation of Akt and ERK survival mechanisms. J Cell Biol 151, 1305-1320. 